\title{
HIGH-RESOLUTION SPECTROSCOPY OF [Ne II] EMISSION FROM AA Tau AND GM Aur*
}

\author{
Joan R. Najita ${ }^{1}$, Greg W. Doppmann ${ }^{1}$, Martin A. Bitner ${ }^{2}$, Matthew J. Richter ${ }^{3}$, John H. Lacy ${ }^{4}$, Daniel T. Jaffe ${ }^{4}$, \\ John S. Carr ${ }^{5}$, Rowin Meijerink ${ }^{6}$, Geoffrey A. Blake ${ }^{6}$, Gregory J. Herczeg ${ }^{7}$, and Alfred E. Glassgold ${ }^{8}$ \\ ${ }^{1}$ National Optical Astronomy Observatory, 950 North Cherry Avenue, Tucson, AZ 85719, USA \\ ${ }^{2}$ Space Telescope Science Institute, 3700 San Martin Drive, Baltimore, MD 21218, USA \\ ${ }^{3}$ Physics Department, University of California at Davis, Davis, CA 95616, USA \\ ${ }^{4}$ Department of Astronomy, University of Texas at Austin, Austin, TX 78712, USA \\ 5 Naval Research Laboratory, Code 7213, Washington, DC 20375, USA \\ ${ }^{6}$ Division of Geological and Planetary Sciences, California Institute of Technology, MS 150-21, Pasadena, CA 91125, USA \\ ${ }^{7}$ Max-Planck-Institut für extraterrestriche Physik, Postfach 1312, D-85741 Garching, Germany \\ ${ }^{8}$ Astronomy Department, University of California, Berkeley, CA 94720, USA \\ Received 2009 January 6; accepted 2009 April 3; published 2009 May 5
}

\begin{abstract}
We present high-resolution $(R=80,000)$ spectroscopy of [Ne II] emission from two young stars, GM Aur and AA Tau, which have moderate to high inclinations. The emission from both sources appears centered near the stellar velocity and is broader than the [Ne II] emission measured previously for the face-on disk system TW Hya. These properties are consistent with a disk origin for the [Ne II] emission we detect, with disk rotation (rather than photoevaporation or turbulence in a hot disk atmosphere) playing the dominant role in the origin of the line width. In the non-face-on systems, the [Ne II] emission is narrower than the $\mathrm{CO}$ fundamental emission from the same sources. If the widths of both diagnostics are dominated by Keplerian rotation, this suggests that the [Ne II] emission arises from larger disk radii on average than does the $\mathrm{CO}$ emission. The equivalent width of the [Ne II] emission we detect is less than that of the spectrally unresolved [Ne II] feature in the Spitzer spectra of the same sources. Variability in the [Ne II] emission or the mid-infrared continuum, a spatially extended [Ne II] component, or a very (spectrally) broad [Ne II] component might account for the difference in the equivalent widths.
\end{abstract}

Key words: circumstellar matter - planetary systems: protoplanetary disks - stars: individual (AA Tau, GM Aur) - stars: pre-main sequence

Online-only material: color figures

\section{INTRODUCTION}

The [Ne II] $12.8 \mu \mathrm{m}$ emission line has been suggested as a potential new probe of the planet formation region of circumstellar disks. It is a potentially powerful diagnostic because the neon in disks is expected to be fully in the gas phase and in atomic form. In addition, the [Ne II] $12.8 \mu \mathrm{m}$ line probes warm, ionized gas, conditions which are believed to characterize the upper atmosphere of the inner disks surrounding classical T Tauri stars (Glassgold et al. 2007; Meijerink et al. 2008) and disk photoevaporative flows (Alexander 2008). Because it is sensitive to low column densities of gas, [Ne II] may also be a useful probe of residual gas surrounding weak-line T Tauri stars or gas in the optically thin regions of transitional disks. Glassgold et al. (2007) predicted that the inner regions $(<20 \mathrm{AU})$ of classical T Tauri disks that are irradiated by stellar X-rays would produce strong [Ne II] emission that could be detected with the Spitzer Space Telescope. Comparably strong [Ne II] emission has indeed been detected, apparently commonly, in Spitzer spectroscopy of T Tauri stars (Pascucci et al. 2007; Lahuis et al. 2007; Ratzka et al. 2007; Espaillat et al. 2007).

While the rough agreement between the predicted and observed [Ne II] line strengths supports the interpretation of a

\footnotetext{
* Based on observations obtained at the Gemini Observatory, which is operated by the Association of Universities for Research in Astronomy, Inc., under a cooperative agreement with the NSF on behalf of the Gemini partnership: the National Science Foundation (United States), the Science and Technology Facilities Council (United Kingdom), the National Research Council (Canada), CONICYT (Chile), the Australian Research Council (Australia), Ministrio da Cincia e Tecnologia (Brazil), and SECYT (Argentina).
}

disk origin for the emission, stronger confirmation can be obtained from a study of resolved [Ne II] line profiles. Herczeg et al. (2007) previously reported high-resolution spectroscopy of [Ne II] emission from TW Hya. TW Hya is a face-on disk system, with an inclination of $i=4^{\circ}$ for the inner disk (Pontoppidan et al. 2008; see also Qi et al. 2004). It is also a "transition object," a T Tauri star whose spectral energy distribution (SED) indicates that the disk continuum is optically thin within a given radius. In the case of TW Hya, modeling of the SED indicates that the disk is optically thin in the continuum $(\lambda>1 \mu \mathrm{m})$ within $\sim 4 \mathrm{AU}$ (Calvet et al. 2002; see also Ratzka et al. 2007).

In their study of the [Ne II] emission from TW Hya, Herczeg et al. (2007) found that the emission was centered at the stellar velocity, consistent with a disk origin. However, the emission was also significantly broader (FWHM $\sim 21 \mathrm{~km} \mathrm{~s}^{-1}$ ) than has been predicted theoretically for a near face-on disk (FWHM $<5 \mathrm{~km} \mathrm{~s}^{-1}$; Meijerink et al. 2008; Glassgold et al. 2007). Herczeg et al. suggested that the observed line width could be explained if the emission arises from (1) a rotating disk at much smaller disk radii $(\sim 0.1 \mathrm{AU})$ than in the model; (2) a disk with a significant turbulent velocity component; or (3) a face-on disk undergoing photoevaporation. While each of these scenarios might account for the observed emission from a faceon system such as TW Hya, Herczeg et al. (2007) proposed that these scenarios could be tested by observing [Ne II] emission from higher inclination disk systems.

A more recent paper (van Boekel et al. 2009) illustrates another possible origin for the [Ne II] emission from $\mathrm{T}$ Tauri stars. High-resolution spectroscopy of the [Ne II] emission from 
the T Tau triplet reveals that the emission is spatially extended and associated with a known outflow in the system. This suggests that [Ne II] emission may arise primarily in outflows rather than disks in systems with strong outflow activity.

To investigate the scenarios proposed by Herczeg et al. (2007) for the origin of [Ne II] emission in T Tauri stars, we present here high-resolution spectroscopy of the [Ne II] emission from AA Tau and GM Aur, T Tauri stars that are not known to show strong outflow activity. Apart from its high inclination $\left(i=75^{\circ}\right.$; Bouvier et al. 1999), AA Tau is a typical classical T Tauri star. GM Aur is an actively accreting transition object that is viewed at an intermediate inclination $\left(i=54^{\circ}\right.$; Simon et al. 2000). The SED of GM Aur indicates that the disk is optically thin in the mid-infrared continuum, suggesting that the disk is devoid of small dust grains in the radial range 5-24 AU (Calvet et al. 2005).

\section{OBSERVATIONS AND DATA REDUCTION}

The observations were carried out using TEXES in its highresolution mode $(R=80,000)$ with a $0{ }^{\prime \prime} 6$ wide slit on the Gemini North telescope on 2007 October 29 and 30 under program ID GN-2007B-C-5. The background sky emission was removed by nodding the source along the slit and subtracting adjacent nod positions. We corrected for telluric absorption with observations of $\alpha \mathrm{CMa}$ obtained at a similar airmass as that of the science targets (the difference in airmass was $<0.2$ ). The spectra were flux calibrated using the low-resolution Spitzer Space Telescope spectra reported by Furlan et al. (2006). Roughly every 10 minutes, we took a series of calibration frames including blank sky and an ambient temperature blackbody. The blackbody observations were used for flat-fielding the data, while the sky emission line observations were used for wavelength calibration. The standard TEXES pipeline (Lacy et al. 2002), which produces wavelength-calibrated one-dimensional spectra, was used to reduce the data. The wavelength solutions have an accuracy in velocity of $\sim 1 \mathrm{~km} \mathrm{~s}^{-1}$.

One challenge associated with these observations is that the [Ne II] line at $12.81 \mu \mathrm{m}$ falls in a gap between two TEXES spectral orders. By tilting a mirror within the instrument, we are able to cover the gap by shifting the spectrum on the detector and thereby accessing the [ $\mathrm{Ne}$ II] line at the long-wavelength end of one order or the short-wavelength end of the next order. In the first case, the blaze efficiency rises toward the blue side of the line, while in the second case, it rises toward the red side of the line.

During the night of October 29, we shifted the optics to enhance the sensitivity on the red side of the [Ne II] line. We spent 2590 s of on-source integration time on GM Aur and 3238 s on AA Tau in this instrument setup. The observed GM Aur line appeared narrow, symmetric, and centered at the stellar velocity, while the emission from AA Tau was broad and redward of the stellar velocity. This led us to search for a line profile component that might be present blueward of the AA Tau stellar velocity on the following night. We spent $2331 \mathrm{~s}$ of on-source integration time on AA Tau on the night of 2007 October 30, with our optics shifted to enhance the sensitivity on the blue side of the [Ne II] line.

The AA Tau data from the two separate nights were combined by interpolating onto a common wavelength scale and, in the spectral regions where data from both nights were available, weighting the contribution from each night. The weighting was used to account for the varying noise across the spectra in the overlap region; the variation resulted primarily from

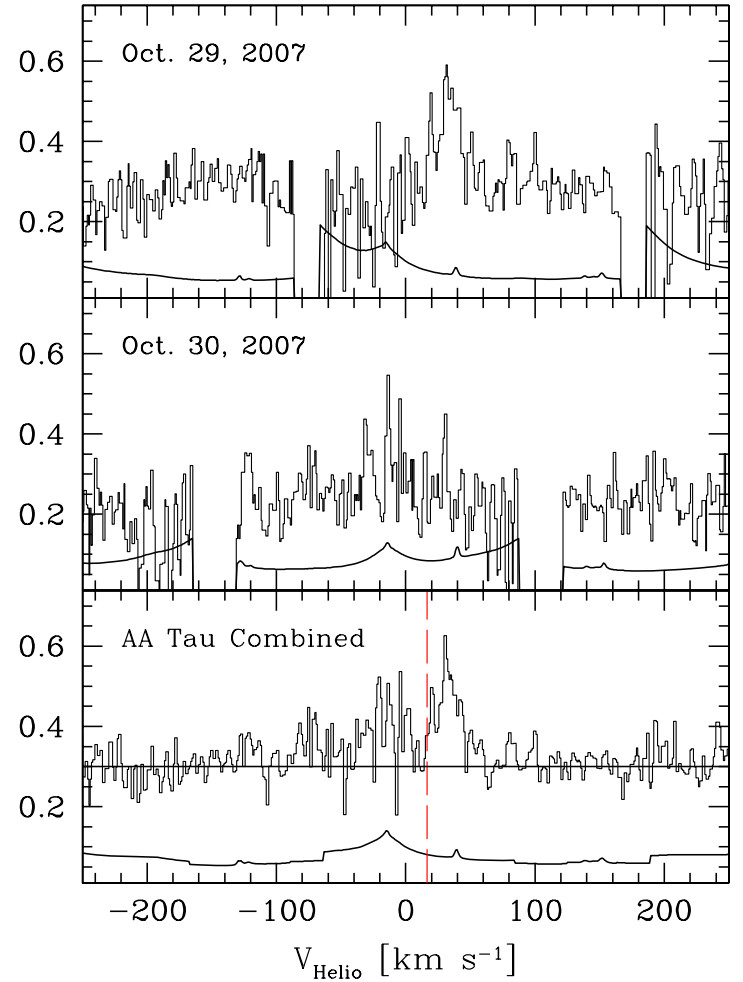

Figure 1. Spectrum of [Ne II] emission from AA Tau (histograms) plotted as a function of heliocentric velocity and smoothed by 3 pixels. The vertical scale is flux density in units of Jy. Spectra obtained on 2007 October 29 and 30 (top and middle panels) are shown along with the combined spectrum (bottom panel). The light lines in the upper two panels indicate the noise per pixel calculated by the TEXES pipeline at each point in the spectrum. The gaps in the spectra in the upper two panels reflect the gaps between the TEXES orders at this wavelength. The vertical dashed line in the bottom panel indicates the stellar velocity.

(A color version of this figure is available in the online journal.)

the rising or falling blaze efficiency. We therefore weighted each data point inversely as the square of the noise, which was calculated assuming that photon noise dominates. This weighting is appropriate if the noise in the two spectra being combined add in quadrature. In practice, the exact value of the weighting factor had little effect on the combined spectrum.

\section{RESULTS}

Figures 1 and 2 show the resulting [Ne II] spectra of AA Tau and GM Aur. The spectra shown have been smoothed by 3 pixels. Because 3 pixels is the width of a spectral resolution element at the wavelength of [Ne II], the smoothing has little impact on our results. Although the larger noise on the blue side of the [Ne II] line in AA Tau makes it difficult to characterize the line profile with certainty, the emission appears to be centered near the stellar heliocentric velocity $\left(v_{\text {helio }}=16.5 \mathrm{~km} \mathrm{~s}^{-1}\right.$; Bouvier et al. 1999) and is broad, extending both redward and blueward of the line center with FWHM $\sim 70 \mathrm{~km} \mathrm{~s}^{-1}$. The line appears approximately double-peaked, with a blueshifted peak possibly further from the stellar velocity and possibly weaker than the redshifted peak. The emission redward of the stellar velocity has an equivalent width of $6.6 \pm 1.4 \AA$.

In comparison, the spectrally unresolved $[\mathrm{Ne}$ II] feature detected in the $R=600$ Spitzer IRS spectrum of AA Tau (Carr \& Najita 2008) has an equivalent width of approximately $24 \AA$. If the emission blueward of the stellar velocity in the TEXES spectrum has an equivalent width equal to that of the redward 


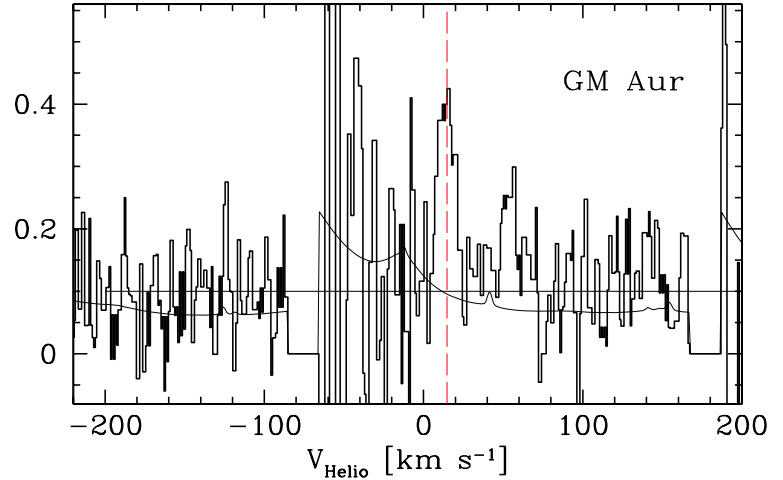

Figure 2. Spectrum of [Ne II] emission from GM Aur plotted as a function of heliocentric velocity and smoothed by 3 pixels. The vertical scale is flux density in units of Jy. The vertical dashed line indicates the stellar velocity, and the horizontal line indicates the continuum level. The light lines indicate the noise per pixel calculated by the TEXES pipeline at each point in the spectrum. In addition to the [Ne II] emission component centered on the stellar velocity, another emission component appears to be present at $+40 \mathrm{~km} \mathrm{~s}^{-1}$ from the stellar velocity.

(A color version of this figure is available in the online journal.)

emission, the total equivalent width (red and blue) is $\sim 0.55$ of the equivalent width seen in the Spitzer spectrum. Such a difference might arise if either the mid-infrared continuum or the $[\mathrm{Ne} \mathrm{II}]$ emission is time variable. Another possibility is that the Spitzer spectrum (4".7 slit width) includes spatially extended [Ne II] emission that is excluded in the narrower slit $\left(0{ }^{\prime \prime} 6\right)$ of the TEXES observation. Spatially extended [Ne II] emission can arise in outflow sources (e.g., Neufeld et al. 2006; van Boekel et al. 2009).

Spectrally unresolved emission from other lines might also enhance the equivalent width of the [Ne II] feature in the Spitzer spectrum. However, known emission features are unlikely to contribute at this wavelength. The synthetic spectrum of AA Tau presented in Carr \& Najita (2008) provides a good fit to the molecular emission detected in the Spitzer spectrum of that source. When examined at high spectral resolution, the synthetic spectrum reveals no strong lines of $\mathrm{H}_{2} \mathrm{O}, \mathrm{HCN}$, or $\mathrm{C}_{2} \mathrm{H}_{2}$ within $200 \mathrm{~km} \mathrm{~s}^{-1}$ of the [Ne II] line. We therefore interpret the emission features detected with TEXES as [Ne II].

The [Ne II] emission from GM Aur has a component of modest width (FWHM $\sim 14 \mathrm{~km} \mathrm{~s}^{-1}$ ) that is centered near the systemic velocity determined from measurements of the surrounding molecular disk $\left(v_{\text {helio }}=14.8 \mathrm{~km} \mathrm{~s}^{-1}\right.$; Dutrey et al. 1998; Simon et al. 2000). There is possible evidence for an additional emission component centered $\sim 40 \mathrm{~km} \mathrm{~s}^{-1}$ redward of the stellar velocity, although it is difficult to be certain because of the limited signal-to-noise ratio of the spectrum. The $40 \mathrm{~km} \mathrm{~s}^{-1}$ component might arise from either infalling gas located close to the star or gas in rotation about the star. In the latter case, we would expect to see an additional emission component with a similar $\sim 40 \mathrm{~km} \mathrm{~s}^{-1}$ velocity offset on the blue side of the line. Because we observed the [ $\mathrm{Ne}$ II] emission in only one order, the GM Aur spectrum has much higher noise on the blue side of the line (see Section 2), and it is impossible to determine whether a corresponding blueshifted component is present. A more complete study of the [Ne II] emission from GM Aur is needed to distinguish between these possibilities.

The emission feature centered near the stellar velocity has an equivalent width of $18 \pm 2 \AA$, and the feature $\sim 40 \mathrm{~km} \mathrm{~s}^{-1}$ redward of the stellar velocity has an equivalent width of $8 \pm 2 \AA$. In comparison, the equivalent width of the spectrally unresolved
[Ne II] feature detected in an $R=600$ Spitzer IRS spectrum of GM Aur (J. R. Najita et al. 2009, in preparation) is approximately $70 \AA$, almost three times the combined equivalent width of the two [Ne II] emission features detected at high spectral resolution. As discussed above, variability in the [Ne II] line or the mid-infrared continuum, alternatively spatially extended [Ne II] emission, might account for the difference in the equivalent widths. We are also insensitive to very broad [Ne II] emission, particularly given the limited signal-to-noise ratio in the continuum. A line 10\% above the marked continuum in Figure 2 and $300 \mathrm{~km} \mathrm{~s}^{-1}$ wide, which is within a Spitzer resolution element, would have an equivalent width of $13 \AA$, similar to the equivalent width reported for the [Ne II] line. Such a broad component would be difficult to detect with our data.

Another possibility is spectrally unresolved emission from other lines. In the Spitzer spectrum of GM Aur, the emission feature detected at the wavelength of [Ne II] is broader than an unresolved line. Perhaps the feature includes a contribution from lines other than [Ne II]. Mid-infrared molecular features such as $\mathrm{H}_{2} \mathrm{O}, \mathrm{HCN}$, or $\mathrm{C}_{2} \mathrm{H}_{2}$ would contribute very negligibly to our TEXES observations, because any such emission features, if present in the Spitzer spectrum of GM Aur, are much weaker than in AA Tau. We therefore interpret the emission features detected with TEXES as [Ne II] for the purpose of this paper.

Thus, as in the case of TW Hya (Herczeg et al. 2007), the [Ne II] line profiles of AA Tau and GM Aur are consistent with emission centered near the stellar velocity. In comparison with the intrinsic (deconvolved) line width of $21 \mathrm{~km} \mathrm{~s}^{-1}$ (FWHM) obtained by Herczeg et al. (2007) for TW Hya, the width of the [Ne II] emission from AA Tau is significantly broader $\left(\sim 70 \mathrm{~km} \mathrm{~s}^{-1}\right)$. While the [Ne II] emission from GM Aur has a velocity component centered on the stellar velocity that is more similar in width $\left(\sim 14 \mathrm{~km} \mathrm{~s}^{-1}\right)$ to the [Ne II] emission from TW Hya, the [Ne II] emission may also include a redshifted emission component that extends to $\gtrsim 50 \mathrm{~km} \mathrm{~s}^{-1}$ of the stellar velocity.

\section{DISCUSSION}

\subsection{Comparison to Profiles of Other Emission Lines}

\subsubsection{Ho Profiles}

The double-peaked shape of the [Ne II] line profile of AA Tau is similar to the shape of the $\mathrm{H} \alpha$ line profile of AA Tau (Bouvier et al. 1999, 2003, 2007; Alencar \& Basri 2000; Edwards et al. 1994). In T Tauri stars, the Balmer lines are believed to form in stellar magnetospheres (e.g., Calvet \& Hartmann 1992; Edwards et al. 1994; Muzerolle et al. 1998). Although both the $\mathrm{H} \alpha$ and [Ne II] profiles of AA Tau are double-peaked, the $\mathrm{H} \alpha$ profile is also much broader (FWHM $\sim 280 \mathrm{~km} \mathrm{~s}^{-1}$; Bouvier et al. 1999).

For GM Aur, the $\mathrm{H} \alpha$ emission is centrally peaked (Edwards et al. 1994), as is the central component of its [Ne II] emission. However, the $\mathrm{H} \alpha$ emission (FWHM of $\sim 220 \mathrm{~km} \mathrm{~s}^{-1}$; Edwards et al. 1994) is also much broader than the [Ne II] emission ( $\sim 14 \mathrm{~km} \mathrm{~s}^{-1} \mathrm{FWHM}$ ); the half-width at zero intensity (HWZI) of the $\mathrm{H} \alpha$ line $\left(\sim 500 \mathrm{~km} \mathrm{~s}^{-1}\right)$ is also much broader than the velocity extent of the redward emission of the [Ne II] emission $\left(\sim 50 \mathrm{~km} \mathrm{~s}^{-1}\right)$. While the $\mathrm{H} \alpha$ and $[\mathrm{Ne}$ II] profiles have some morphological similarities, the difference in the velocity widths suggests that the $[\mathrm{Ne}$ II] emission arises primarily from a different region than the stellar magnetosphere. 


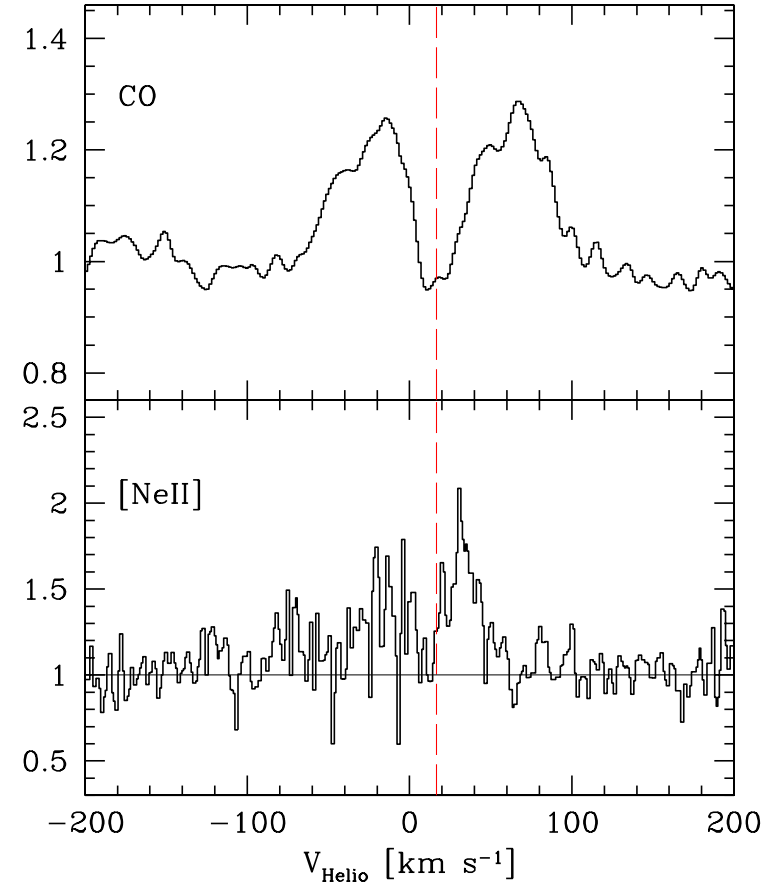

Figure 3. Top: CO emission profile from AA Tau in the $4.9 \mu \mathrm{m}$ region, normalized to the continuum. The vertical dashed line indicates the stellar velocity $\left(v_{\text {helio }}=16.5 \mathrm{~km} \mathrm{~s}^{-1}\right)$. The line profile shown is the average of the $v=$ 1-0 P25 through P28 lines. Regions of poor telluric correction were excluded from the average. Bottom: [Ne II] line profile of AA Tau from the combined spectrum shown in Figure 1, normalized to the continuum.

(A color version of this figure is available in the online journal.)

\subsection{2. $\mathrm{CO}$ Fundamental and $\mathrm{UV} \mathrm{H}_{2}$ Profiles}

The CO fundamental lines from AA Tau are also doublepeaked, similar to the shape of the [Ne II] line in AA Tau. CO fundamental emission is believed to probe the inner regions of T Tauri disks (see Najita et al. 2007a for a review). Figure 3 compares the [Ne II] profile of AA Tau with the average line profile of $\mathrm{CO}$ emission from AA Tau that was obtained by J. Carr, J. Najita, and N. Crockett on the night of 2004 November 26 using NIRSPEC on the Keck II telescope on Mauna Kea. The line profile shown is the average of four $v=1-0$ lines in the $4.9 \mu \mathrm{m}$ region. Regions of poor telluric correction $(\lesssim 85 \%$ transmission, as determined from observations of the telluric standard) were excluded from the average. The double-peaked CO line profile has a FWHM of $\sim 140 \mathrm{~km} \mathrm{~s}^{-1}$ and a peak-topeak separation of $\sim 80 \mathrm{~km} \mathrm{~s}^{-1}$. Thus, the CO profile is broader than the $[\mathrm{Ne}$ II] line profile, but narrower than the Balmer line profile. A preliminary analysis of the basic properties of the $\mathrm{CO}$ fundamental emission from AA Tau finds that the emission is optically thick and has an average temperature of $\sim 900 \mathrm{~K}$ (Carr \& Najita 2008). A more detailed analysis of the CO emission will be presented in a future publication.

CO fundamental emission has been reported from GM Aur by Salyk et al. (2007), although it was not detected in an earlier study (Najita et al. 2003). Because of the low infrared continuum excess of GM Aur compared to that of AA Tau (GM Aur is a transition object), the stellar photosphere makes a more significant contribution to the GM Aur spectrum than it does in the case of AA Tau. We therefore first corrected for stellar photospheric absorption in the Salyk et al. (2007) spectrum before constructing the average $\mathrm{CO}$ line profile. Following the approach described in Najita et al. (2008), we created a synthetic stellar spectrum that is appropriate for the stellar spectral type

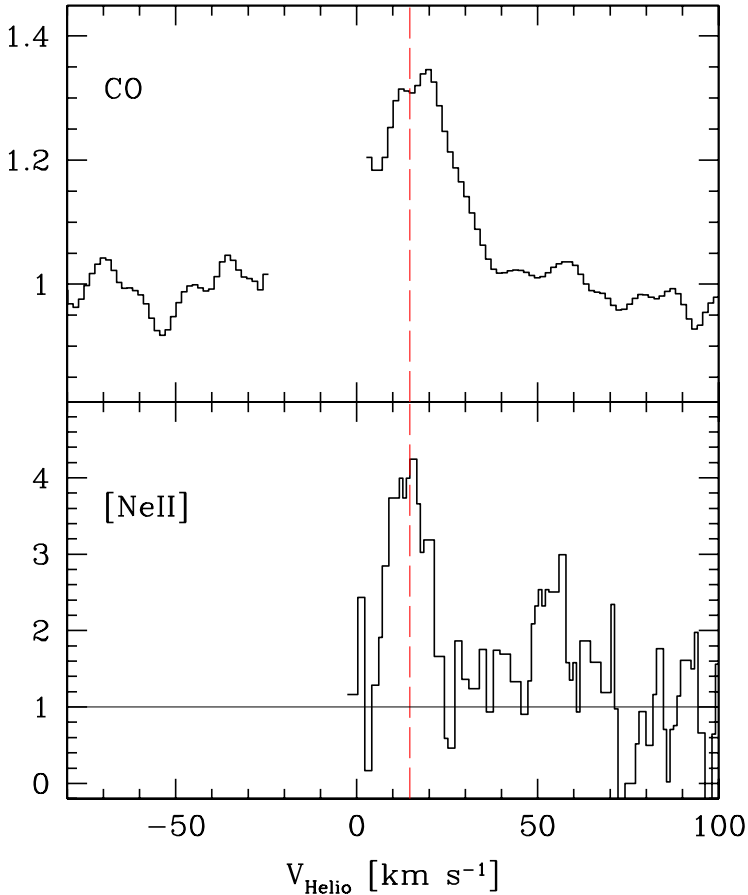

Figure 4. Top: $\mathrm{CO}$ emission profile from GM Aur in the $4.7 \mu \mathrm{m}$ region, normalized to the continuum. The vertical dashed line indicates the stellar velocity $\left(v_{\text {helio }}=14.7 \mathrm{~km} \mathrm{~s}^{-1}\right)$. The line profile shown is the average of the $v=1-0 \mathrm{P} 8$ through $\mathrm{P} 12$ lines. In constructing the profile, the stellar photospheric contribution to the spectrum was first removed and regions of poor telluric correction were excluded from the average. Bottom: [Ne II] line profile of GM Aur from the spectrum shown in Figure 2, normalized to the continuum.

(A color version of this figure is available in the online journal.)

of the source (K3V; Herbig \& Bell 1988). We also used a stellar rotational velocity of $v \sin i=8 \mathrm{~km} \mathrm{~s}^{-1}$ (see also Hartmann et al. 1986). We found a good fit to the observed structure in the continuum (i.e., in the region away from the $1-0$ CO lines) with a veiling of 1 at $4.7 \mu \mathrm{m}$. This value for the veiling at $4.7 \mu \mathrm{m}$ is consistent with that implied by the SED of GM Aur (Furlan et al. 2006). We subtracted the veiled stellar continuum from the spectrum, and we then added back an equivalent (featureless) continuum in order to show the strength of the emission relative to the continuum.

Figure 4 compares the [Ne II] profile of GM Aur with the average $\mathrm{CO}$ line profile constructed from the resulting spectrum in the region of the $v=1-0$ P8 through P12 transitions. Regions of poor telluric correction $(\lesssim 75 \%$ transmission) were excluded from the average. The average $\mathrm{CO}$ line profile differs from that reported by Salyk et al. (2007) in that there is no significant central dip in the average line profile, because the stellar photospheric contribution to the line profile has been removed. Because the average line profile is now more centrally peaked, the FWHM of the average line profile $\left(\sim 30 \mathrm{~km} \mathrm{~s}^{-1}\right)$ is also smaller than the value of $\sim 50 \mathrm{~km} \mathrm{~s}^{-1}$ reported by Salyk et al. (2007).

The $[\mathrm{Ne}$ II] and $\mathrm{CO}$ line properties can also be compared with those of UV fluorescent $\mathrm{H}_{2}$ emission, another line diagnostic that is believed to probe the conditions in inner circumstellar disks (e.g., Herczeg et al. 2006 and references therein; see, e.g., Najita et al. 2007a for a review). In the case of TW Hya, Herczeg et al. (2007) found that the [Ne II] emission width $\left(21 \mathrm{~km} \mathrm{~s}^{-1}\right)$ is broader than the $\mathrm{UV} \mathrm{H}_{2}$ emission $\left(14 \mathrm{~km} \mathrm{~s}^{-1}\right)$, which is broader than the $\mathrm{CO}$ fundamental emission $\left(\sim 8 \mathrm{~km} \mathrm{~s}^{-1}\right.$; Salyk et al. 2007). A similar decreasing sequence is inferred for the nominal temperatures of the emitting gas that gives 
Table 1

[Ne II], $\mathrm{H}_{2}$, and CO Line FWHM

\begin{tabular}{lccccc}
\hline \hline Source & $\begin{array}{c}i \\
(\mathrm{deg})\end{array}$ & $\begin{array}{c}\sigma(\mathrm{Ne} \mathrm{II}) \\
\left(\mathrm{km} \mathrm{s}^{-1}\right)\end{array}$ & $\begin{array}{c}\sigma\left(\mathrm{H}_{2}\right) \\
\left(\mathrm{km} \mathrm{s}^{-1}\right)\end{array}$ & $\begin{array}{c}\sigma(\mathrm{CO}) \\
\left(\mathrm{km} \mathrm{s}^{-1}\right)\end{array}$ & References \\
\hline AA Tau & 75 & 70 & $\ldots$ & 145 & B99, Here \\
GM Aur & 54 & 14 & $\ldots$ & 30 & S00, S07, Here \\
TW Hya & 4 & 21 & 14 & 8 & P08, H07, S07 \\
BP Tau & $<50$ & $\ldots$ & 57 & 70 & A02, N03 \\
DF Tau & 80 & $\ldots$ & 27 & 65 & A02, N03 \\
& & & 23 & & H06 \\
RW Aur & 40 & $\ldots$ & 52 & $80-250$ & A02, N03 \\
V836 Tau & 65 & $\ldots$ & 24 & 130 & H06, N08 \\
\hline
\end{tabular}

References. A02, Ardila et al. (2002); B99, Bouvier et al. (1999); H06, Herczeg et al. (2006); H07, Herczeg et al. (2007); N03, Najita et al. (2003); N08, Najita et al. (2008); P08, Pontoppidan et al. (2008); S00, Simon et al. (2000); S07, Salyk et al. (2007).

rise to these features: $\sim 4000 \mathrm{~K}$ for [Ne $\mathrm{II}$ ] (Glassgold et al. 2007), $2000 \mathrm{~K}$ for $\mathrm{H}_{2}$ (Herczeg et al. 2004), and $\sim 800 \mathrm{~K}$ for CO (Salyk et al. 2007). Such a progression might occur if disk atmospheres experience transonic turbulence, which is enhanced in the warmer, atomic regions higher up in the disk atmosphere. While this interpretation might be considered for a face-on system such as TW Hya, the progression of line widths in TW Hya, from broader [Ne II] lines to narrower $\mathrm{H}_{2}$ and CO lines, would not be expected to hold in higher inclination systems where disk rotation would play a larger role.

Indeed, for AA Tau, we find that the width of the CO emission is instead broader than the [Ne II] emission. For GM Aur, the CO line $\left(\mathrm{FWHM} \sim 30 \mathrm{~km} \mathrm{~s}^{-1}\right)$ is also broader than the central component of the $\left[\mathrm{Ne}\right.$ II] line (FWHM $\sim 14 \mathrm{~km} \mathrm{~s}^{-1}$ ); the HWZI of the average $\mathrm{CO}$ line profile could be as large as $\sim 50 \mathrm{~km} \mathrm{~s}^{-1}$ (Salyk et al. 2007), comparable to the velocity extent of the red component of the $[\mathrm{Ne}$ II $]$ emission $\left(\sim 50 \mathrm{~km} \mathrm{~s}^{-1}\right)$. Thus, the $\mathrm{CO}$ emission is broader or comparable to the width of the [Ne II] emission in the two sources studied. If both diagnostics are dominated by disk rotation, this suggests that the [Ne II] emission arises from larger disk radii on average than the $\mathrm{CO}$.

This is consistent, in general, with models of the ionization and thermal structure of T Tauri disks (e.g., Glassgold et al. 2004, 2007; Meijerink et al. 2008). In the Glassgold et al. models, the surface of the disk is heated and ionized by stellar X-rays, producing a low column density $\left(N_{\mathrm{H}} \sim 10^{20} \mathrm{~cm}^{-2}\right)$ surface layer of hot $(\sim 4000 \mathrm{~K})$ gas that extends to $\gtrsim 10 \mathrm{AU}$. Significant $[\mathrm{Ne}$ II] is found to emerge from the disk surface region within 20 AU (Glassgold et al. 2007; Meijerink et al. 2008). In contrast, $\mathrm{CO}$ becomes abundant at much larger disk vertical column densities $\left(N_{\mathrm{H}}>10^{20} \mathrm{~cm}^{-2}\right)$, and the warm temperatures and high densities needed to produce the $\mathrm{CO}$ emission ( $>500 \mathrm{~K}$ ) likely limit the emission to smaller disk radii, within a few AU.

Although for TW Hya the width of the $\mathrm{UV} \mathrm{H}_{2}$ emission is broader than the $\mathrm{CO}$ fundamental emission, the inverse is true for other sources with $\mathrm{UV} \mathrm{H}_{2}$ and $\mathrm{CO}$ fundamental emission line widths that have been reported in the literature (see Table 1). Thus, as might be expected, the progression of line widths found for TW Hya among the [Ne II], $\mathrm{H}_{2}$, and $\mathrm{CO}$ emission lines does not appear to be typical of $\mathrm{T}$ Tauri stars.

\subsection{Origin of [Ne II] Emission}

Herczeg et al. (2007) offered three possible interpretations for the origin of the width of the [Ne II] line observed from TW Hya: (1) Keplerian rotation from the very inner disk region, (2) transonic turbulence in a $\sim 10,000 \mathrm{~K}$ disk atmosphere, or (3) photoevaporation at $\sim 10 \mathrm{~km} \mathrm{~s}^{-1}$ from both faces of a face-on gaseous disk that is optically thin in the mid-infrared continuum. While each of these explanations could, in principle, account for the observed $\sim 21 \mathrm{~km} \mathrm{~s}^{-1}$ line width of TW Hya given its low inclination, they would produce different line profiles for systems viewed at higher inclinations.

At higher inclination, a line profile dominated by disk rotation would remain symmetric about the stellar velocity but would become broader. In comparison, a line profile dominated by turbulence would show little difference when viewed at higher inclination. In the case of a photoevaporative flow from a disk that is optically thick in the mid-infrared continuum, the disk would occult the receding flow, producing a line profile that is blueshifted by up to $\sim 10 \mathrm{~km} \mathrm{~s}^{-1}$, the nominal velocity of a photoevaporative flow (e.g., Font et al. 2004).

A physical picture analogous to this last scenario is invoked to account for the high-velocity blueshifted components of the [O I] $6300 \AA$ line emission from T Tauri stars (e.g., Edwards et al. 1993), although the high velocities observed in the [O I] case indicate a dynamical (rather than thermal) ejection process. Font et al. (2004) studied whether a lower velocity thermally driven disk photoevaporative flow could account for the properties of the low-velocity component of the [O I] emission. A more recent paper explores the disk photoevaporative flow hypothesis specifically for [Ne II], in both the case of a continuous disk and a disk with an inner hole (Alexander 2008). In a system viewed edge-on, the [Ne II] line profile is predicted to be dominated by Keplerian disk rotation and to be centered on the stellar velocity. At lower inclinations, the [Ne II] line profile is predicted to be blueshifted from the stellar velocity by $5-10 \mathrm{~km} \mathrm{~s}^{-1}$.

Of the three explanations invoked to explain the width of the [Ne II] line of TW Hya, only disk rotation could plausibly account for the broad profile of the [Ne II] emission from AA Tau. The width of the emission greatly exceeds the width expected from either disk turbulence or photoevaporation. The apparent double-peaked line profile might indicate that the emission arises from a limited range of disk radii, similar to the interpretation given to the double-peaked $\mathrm{CO}$ overtone lines in sources such as WL16 (Carr et al. 1993; Najita et al. 1996). For Keplerian rotation about a $0.7 M_{\odot}$ star viewed at an inclination of $75^{\circ}$, the redward velocity extent of the [Ne II] emission and the redward emission peak (to $+45 \mathrm{~km} \mathrm{~s}^{-1}$ and at $\sim 18 \mathrm{~km} \mathrm{~s}^{-1}$ from the stellar velocity, respectively) would indicate emission extending from an inner radius of $\sim 0.3 \mathrm{AU}$ out to an outer radius of $\sim 1.8$ AU.

The narrow $\left(\sim 14 \mathrm{~km} \mathrm{~s}^{-1}\right.$ FWHM) component of the [Ne II] emission from GM Aur that is centered at the stellar velocity could plausibly be explained by turbulent broadening. It might also arise from a rotating Keplerian disk at radii beyond several AU given the stellar mass $\left(0.84-1.0 M_{\odot}\right)$ and inclination $\left(i=54^{\circ}\right)$ measured for the system (Simon et al. 2000; Dutrey et al. 2008). The lack of a velocity shift of this component from the stellar velocity argues against photoevaporation playing a significant role in the emission (compare with Font et al. 2004; Alexander 2008). The high velocity of the redshifted component could plausibly be accounted for by disk rotation if an (undetected) blueshifted component is also present. The $\sim 40 \mathrm{~km} \mathrm{~s}^{-1}$ velocity shift measured for this component would correspond to gas in Keplerian rotation at an orbital radius of $0.3 \mathrm{AU}$.

In comparison, Glassgold et al. (2007) predicted that an Xray irradiated disk atmosphere would produce [Ne II] emission 


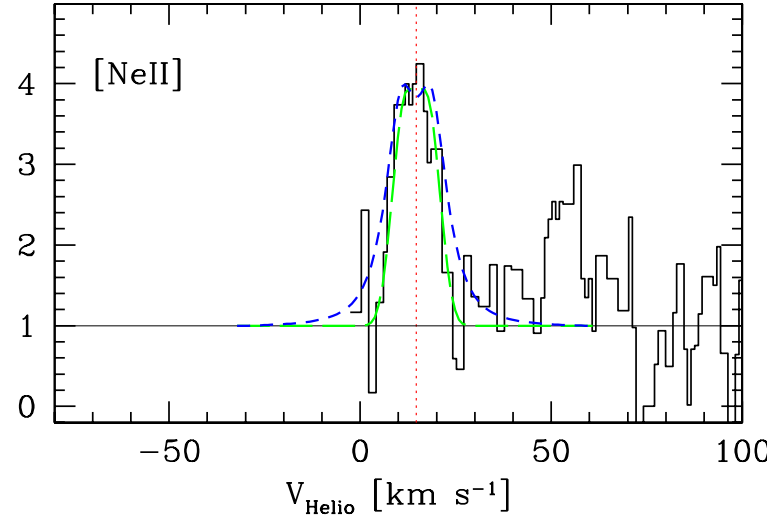

Figure 5. [Ne II] emission profile of GM Aur, compared with synthetic [Ne II] disk emission profiles from an X-ray irradiated disk (Meijerink et al. 2008; see the text for details). The model profiles assume Keplerian rotation and the stellar mass $\left(0.85 M_{\odot}\right)$ and inclination $\left(i=54^{\circ}\right)$ of GM Aur reported by Simon et al (2000). The emission extends from an outer radius of $\sim 25 \mathrm{AU}$, beyond which the [Ne II] emission is insignificant, inward to $0.25 \mathrm{AU}$ (short dashed blue line) or $4 \mathrm{AU}$ (long dashed green line). A vertical scaling factor is applied to each model to facilitate comparison with the observed profile.

(A color version of this figure is available in the online journal.)

from radii within $\sim 20 \mathrm{AU}$. [Ne II] emission is expected to arise from a similar range of disk radii in the case of a disk irradiated by stellar UV photons (Gorti \& Hollenbach 2008; Alexander 2008). In an updated study that extends the Glassgold et al. (2007) calculation inward to 0.25 AU, Meijerink et al. (2008) showed the predicted [Ne II] emissivity per unit radius, $P$, on a velocity scale $v_{\mathrm{K}}$, where $v_{K}$ is the Keplerian rotational velocity at a given radius. This radial contribution function corresponds to a [Ne II] line profile with a FWHM approximately equal to $0.75 v_{1} \sin i$, where $v_{1}$ is the Keplerian velocity at $1 \mathrm{AU}$. The line width is smaller than the width of the $P\left(v_{\mathrm{K}}\right)$ distribution because the line profile accounts for how the flux from a given annulus, when viewed at an inclination $i$, is distributed over a range of velocities between 0 and $v_{\mathrm{K}}$.

For the stellar mass and inclination of GM Aur, the width of the [Ne II] line is expected to be $17 \mathrm{~km} \mathrm{~s}^{-1}$, similar to the observed $14 \mathrm{~km} \mathrm{~s}^{-1}$ width of the central [Ne II] emission component of GM Aur. Figure 5 compares the observed [Ne II] profile of GM Aur with a model profile constructed from the $P\left(v_{\mathrm{K}}\right)$ distribution of Meijerink et al. (2008), where the emission extends from an outer radius of $\sim 25 \mathrm{AU}$, beyond which the [Ne II] emission is insignificant, in to the minimum radius of $0.25 \mathrm{AU}$ studied by those authors (short dashed blue line). The redshifted [Ne II] emission component extends to higher velocities, $\sim 40 \mathrm{~km} \mathrm{~s}^{-1}$, and is not well accounted for by the model profile. For the $i=75^{\circ}$ inclination of AA Tau, the Meijerink et al. (2008) results would suggest a width for the [Ne II] line of $15-18 \mathrm{~km} \mathrm{~s}^{-1}$ for a stellar mass of $0.5-0.7 \mathrm{M}_{\odot}$. The observed width of the [Ne II] emission from AA Tau is significantly broader than this value.

The difference between the observed profiles and the theoretical predictions may be due to the simple physical picture adopted in the disk emission models; real systems are likely to be more complex. Glassgold et al. (2004) adopted the conventional (flared, azimuthally symmetric) disk geometry of D'Alessio et al. (1999). In contrast, in AA Tau the modulation of its photometric and spectroscopic properties suggest that the inner disk edge (near the corotation radius) has a large-scale height over a range in azimuth (Bouvier et al. 1999, 2003, 2007). Periodic variations in the optical light curve occur in phase with enhanced redshifted absorption in the Balmer line profiles. These and other observed properties have been interpreted as evidence for an optically thick occulting screen produced by a magnetically warped dusty inner disk edge. In a system with an inflated inner disk edge, more of the stellar ionizing photons (X-rays or UV) may be deposited close to the star, resulting in [Ne II] emission from smaller radii and higher disk rotational velocities.

GM Aur is also an unusual source in that, like TW Hya, it is a transition object. Based on the shape of the SED, Calvet et al. (2005) estimate that the disk is devoid of dust in the radial range 5-24 AU, with a small amount of dust present within 5 AU. Earlier studies of the SED found a smaller optically thin region out to 4 AU (Rice et al. 2003; see also Bergin et al. 2004). These authors speculate that the optically thin region arises from the presence of a companion that has carved out a gap in the disk (see also Marsh \& Mahoney 1992).

In comparison, the [Ne II] emission component centered on the systemic velocity in GM Aur has a HWZI of $\sim 10 \mathrm{~km} \mathrm{~s}^{-1}$. If the width of the emission arises from Keplerian rotation, the emission arises from $\gtrsim 4 \mathrm{AU}$, given the stellar mass and inclination of GM Aur (Dutrey et al. 1998; Simon et al. 2000). This component may therefore arise from within the optically thin region (5-24 AU) in the interpretation of the SED favored by Calvet et al. (2005; see also Dutrey et al. 2008), or from the inner region of the outer disk, if the outer disk extends in to the smaller radii favored by Rice et al. (2003). Figure 5 shows a model profile constructed using the $P\left(v_{\mathrm{K}}\right)$ distribution of Meijerink et al. (2008) where the emission extends in to $4 \mathrm{AU}$ (long dashed green line). Higher signal-to-noise ratio data are needed to determine if this model profile provides a better fit than one in which the inner radius extends in to much smaller radii (e.g., $0.25 \mathrm{AU}$; short dashed blue line).

The redward component of the [Ne II] emission extends to $\sim 33-55 \mathrm{~km} \mathrm{~s}^{-1}$ from the stellar velocity. If the [Ne II] line profile is symmetric across the stellar velocity and arises from Keplerian rotation, the redward emission component can be inferred to arise from disk radii 0.16-0.5 AU. This component may represent emission from a gaseous inner disk close to the star. Such a gaseous disk is expected to be present since GM Aur experiences continued stellar accretion at a rate typical of T Tauri stars $\left(\sim 10^{-8} M_{\odot} \mathrm{yr}^{-1}\right.$; Najita et al. 2007b).

\section{SUMMARY AND FUTURE DIRECTIONS}

The observations reported here bring to a grand total of four the number of T Tauri [Ne II] emission sources that have been studied at high spectral resolution. In the high accretion rate system $\mathrm{T}$ Tau, the $[\mathrm{Ne} \mathrm{II}]$ emission is associated primarily with outflow activity (van Boekel et al. 2009). In the three lower accretion rate cases studied thus far (TW Hya, AA Tau, GM Aur), the [Ne II] emission appears to be centered at the stellar velocity, consistent with a disk origin for the emission. The [Ne II] emission is broader in the non-face-on systems (AA Tau, GM Aur) than in the face-on TW Hya, a result that is also consistent with a disk origin for the emission.

In the non-face-on systems, the [Ne II] emission width is narrower than (or comparable to) the $\mathrm{CO}$ emission width. If the widths of both diagnostics are dominated by Keplerian rotation, this suggests that the [Ne II] emission arises from larger disk radii on average than the $\mathrm{CO}$ emission. This is consistent, in general, with models of the ionization and thermal structure of T Tauri disks (e.g., Glassgold et al. 2007; Meijerink et al. 2008).

The observed [Ne II] line profiles show little evidence for an origin in a disk photoevaporative flow. This is particularly 
so in the case of GM Aur, where the central component of the [Ne II] emission is narrow enough to plausibly arise in a photoevaporative flow, but the profile is centered at the stellar velocity rather than showing the $5-10 \mathrm{~km} \mathrm{~s}^{-1}$ blueshift that is predicted for emission arising in a photoevaporative flow (Alexander 2008). Photoevaporative flows may be present in these systems but contribute a small fraction of the [Ne II] emission. More recent work by Pascucci \& Sterzik (2009) finds stronger evidence for photoevaporative flows in other [Ne II]emitting systems.

The equivalent width of the [Ne II] emission we detect is less than that of the spectrally unresolved [Ne II] feature in the Spitzer spectra of the same sources. Variability in the [Ne II] emission or the mid-infrared continuum, a spatially extended [Ne II] component, or a very (spectrally) broad [ $\mathrm{Ne}$ II] component might account for the difference in the equivalent widths. Further work is needed to understand the origin of this discrepancy.

These results illustrate the ability of high-resolution spectroscopy to probe the origin of the [Ne II] emission from $\mathrm{T}$ Tauri stars. Further measurements of resolved [Ne II] line profiles, for both classical $\mathrm{T}$ Tauri stars and transition objects, are needed to determine whether the results obtained here apply to the majority of [Ne II]-emitting $\mathrm{T}$ Tauri stars. In addition, higher sensitivity line profiles than those reported here would be useful to probe the origin of the [Ne II] emission in detail.

We thank Nathan Crockett for his help reducing the CO spectrum of AA Tau, and Colette Salyk for making available her published CO spectrum of GM Aur. We thank the Gemini staff for their support of TEXES observations on Gemini North. The development of TEXES was supported by grants from the NSF and the NASA/USRA SOFIA project. Modification of TEXES for use on Gemini was supported by Gemini Observatory. Observations with TEXES were supported by NSF grant AST-0607312. Financial support for the work of J.R.N. and G.W.D. was provided by the NASA Origins of Solar Systems program (NNH07AG51I) and the NASA Astrobiology Institute under Cooperative Agreement CAN-02-OSS-02 issued through the Office of Space Science. This work was also supported by the Life and Planets Astrobiology Center (LAPLACE). Basic research in infrared astronomy at the Naval Research Laboratory is supported by 6.1 base funding. M.J.R. acknowledges support from NSF grant AST-0708074 and NASA grant NNG04GG92G. This work is based on observations obtained at the Gemini Observatory, which is operated by the Association of Universities for Research in Astronomy, Inc., under a cooperative agreement with the NSF on behalf of the Gemini partnership: the National Science Foundation (United States), the Particle Physics and Astronomy Research Council (United Kingdom), the National Research Council (Canada), CONICYT (Chile), the Australian Research Council (Australia), CNPq (Brazil), and CONICET (Argentina). The NIRSPEC data presented herein were obtained at the W. M. Keck Observatory, in part via the TSIP program administered by NOAO and in part from telescope time allocated to NASA through the agency's scientific partnership with the California Institute of Technology and the University of California. The Observatory was made possible by the generous financial support of the W. M. Keck Foundation. The authors recognize and acknowledge the very significant cultural role and reverence that the summit of Mauna Kea has always had within the indigenous Hawaiian commu- nity. We are most fortunate to have the opportunity to conduct observations from this mountain.

Facilities: Gemini (TEXES), Keck (NIRSPEC).

\section{REFERENCES}

Alencar, S. H. P., \& Basri, G. 2000, AJ, 119, 1881

Alexander, R. D. 2008, MNRAS, 391, L64

Ardila, D. R., Basri, G., Walter, F. M., Valenti, J. A., \& Johns-Krull, C. M. 2002, ApJ, 566, 1100

Bergin, E., et al. 2004, ApJ, 614, L133

Bouvier, J., et al. 1999, A\&A, 349, 619

Bouvier, J., et al. 2003, A\&A, 409, 169

Bouvier, J., et al. 2007, A\&A, 463, 1017

Calvet, N., D’Alessio, P., Hartmann, L., Wilner, D., Walsh, A., \& Sitko, M. 2002, ApJ, 568, 1008

Calvet, N., \& Hartmann, L. 1992, ApJ, 386, 239

Calvet, N., et al. 2005, ApJ, 630, L185

Carr, J. S., \& Najita, J. R. 2008, Science, 319, 1504

Carr, J. S., Tokunaga, A. T., Najita, J., Shu, F. H., \& Glassgold, A. E. 1993, ApJ, 411, L37

D’Alessio, P., Calvet, N., Hartmann, L., Lizano, S., \& Cantó, J. 1999, ApJ, 527, 893

Dutrey, A., Guilloteau, S., Prato, L., Simon, M., Duvert, G., Schuster, K., \& Menard, F. 1998, A\&A, 338, L63

Dutrey, A., et al. 2008, A\&A, 490, L15

Edwards, S., Hartigan, P., Ghandour, L., \& Andrulis, C. 1994, AJ, 108, 1056

Edwards, S., Ray, T., \& Mundt, R. 1993, in Protostars and Planets III, ed. E. Levy \& J. Lunine (Tucson, AZ: Univ. Arizona Press), 567

Espaillat, C., et al. 2007, ApJ, 664, L111

Font, A. S., McCarthy, I. G., Johnstone, D., \& Ballantyne, D. R. 2004, ApJ, 607,890

Furlan, E., et al. 2006, ApJS, 165, 568

Glassgold, A. E., Najita, J., \& Igea, J. 2004, ApJ, 615, 972

Glassgold, A. E., Najita, J. R., \& Igea, J. 2007, ApJ, 656, 515

Gorti, U., \& Hollenbach, D. 2008, ApJ, 683, 287

Hartmann, L., Hewett, R., Stahler, S., \& Mathieu, R. D. 1986, ApJ, 309, 275

Herbig, G. H., \& Bell, K. R. (ed.) 1988, Third Catalog of Emission-Line Stars of the Orion Population, Lick Observatory Bulletin (Santa Cruz: Lick Observatory)

Herczeg, G. J., Linsky, J. L., Walter, F. M., Gahm, G. F., \& Johns-Krull, C. M. 2006, ApJS, 165, 256

Herczeg, G. J., Najita, J. R., Hillenbrand, L. A., \& Pascucci, I. 2007, ApJ, 670 , 509

Herczeg, G. J., Wood, B. E., Linsky, J. L., Valenti, J. A., \& Johns-Krull, C. M. 2004, ApJ, 607, 369

Lacy, J. H., Richter, M. J., Greathouse, T. K., Jaffe, D. T., \& Zhu, Q. 2002, PASP, 114,153

Lahuis, F., van Dishoeck, E. F., Blake, G. A., Evans, N. J., II, Kessler-Silacci, J. E., \& Pontoppidan, K. M. 2007, ApJ, 665, 492

Marsh, K. A., \& Mahoney, M. J. 1992, ApJ, 395, L115

Meijerink, R., Glassgold, A. E., \& Najita, J. R. 2008, ApJ, 676, 518

Muzerolle, J., Hartmann, L., \& Calvet, N. 1998, AJ, 116, 455

Najita, J., Carr, J. S., Glassgold, A. E., Shu, F. H., \& Tokunaga, A. T. 1996, ApJ, 462,919

Najita, J. R., Carr, J. S., Glassgold, A. E., \& Valenti, J. A. 2007a, in Protostars and Planets V, ed. B. Reipurth, D. Jewitt, \& K. Keil (Tucson, AZ: Univ. Arizona Press), 507

Najita, J., Carr, J. S., \& Mathieu, R. D. 2003, ApJ, 589, 931

Najita, J. R., Crockett, N., \& Carr, J. S. 2008, ApJ, 687, 1168

Najita, J. R., Strom, S. E., \& Muzerolle, J. 2007b, MNRAS, 378, 369

Neufeld, D. A., et al. 2006, ApJ, 649, 816

Pascucci, I., \& Sterzik, M. 2009, ApJ, submitted

Pascucci, I., et al. 2007, ApJ, 663, 383

Pontoppidan, K. M., Blake, G. A., van Dishoeck, E. F., Smette, A., Ireland, M. J., \& Brown, J. 2008, ApJ, 684, 1323

Qi, C., et al. 2004, ApJ, 616, L11

Ratzka, T., Leinert, C., Henning, T., Bouwman, J., Dullemond, C. P., \& Jaffe, W. 2007, A\&A, 471, 173

Rice, W. K. M., Wood, K., Armitage, P. J., Whitney, B. A., \& Bjorkman, J. E. 2003, MNRAS, 342, 79

Salyk, C., Blake, G. A., Boogert, A. C. A., \& Brown, J. M. 2007, ApJ, 655, L105

Simon, M., Dutrey, A., \& Guilloteau, S. 2000, ApJ, 545, 1034

van Boekel, R., Güdel, M., Henning, Th., Lahuis, F., \& Pantin, E. 2009, A\&A, 497, 137 